\title{
Parachute mitral valve assessed by cardiac magnetic resonance
}

\author{
GRAZIA CASAVECCHIA $^{1}$, MATTEO GRAVINA ${ }^{1}$, STEFANO ZICCHINO $^{1}$, SILVANA CAPALBO $^{1}$, \\ MATTEO DI BIASE ${ }^{2}$, NATALE DANIELE BRUNETTI ${ }^{1, *}$ \\ ${ }^{1}$ Department of Medical and Surgical Sciences, University of Foggia, Foggia, Italy \\ ${ }^{2}$ GVM Care \& Research, Bari, Italy \\ *Corresponding author: Natale Daniele Brunetti, MD, PhD, FESC; Department of Medical and Surgical Sciences, University of Foggia, \\ Viale Pinto 1, 71122 Foggia, Italy; Phone: +39 3389 112358; Fax: +39 0881 745424; E-mail: natale.brunetti@unifg.it
}

(Received: November 14, 2018; Revised manuscript received: December 28, 2018; Accepted: January 28, 2019)

\begin{abstract}
Parachute mitral valve (PMV) is a congenital cardiac malformation where typically all tendons chordae converge and insert into one major papillary muscle. We report the case of 27-year-old man affected by Hodgkin's lymphoma-mixed cellularity subtype with polychemotherapy treatment and Barrett's esophagus who came to our attention for routine echocardiographic exam and evaluation of left ventricular function. In the presence of normal left ventricular systolic function and mild-moderate mitral regurgitation, a careful examination of mitral apparatus showed a single papillary muscle suspected for PMV. In the presence of Barrett's esophagus, the patient was further assessed cardiac magnetic resonance (CMR) that confirmed the diagnosis of PMV and excluded other associated congenital anomalies. Despite the rare incidence of PMV and a diagnosis generally based on transesophageal echocardiography, CMR could represent a useful tool, in selected cases, to confirm the diagnosis of PMV, identify other possible congenital anomalies, and assess ventricular and valvular function.
\end{abstract}

Keywords: parachute mitral valve, cardiac magnetic resonance, valve disease, echocardiography, imaging

\section{Background}

Parachute mitral valve (PMV) is a congenital cardiac malformation where typically all chordae tendineae converge and insert into one major papillary muscle. This malformation also grouped the cases of two papillary muscles that are too close creating the same hemodynamic effect of single muscle $[1,2]$ or where there is a dominant papillary muscle with its tip reaching to the valve leaflets generally named as parachute-like asymmetric mitral valve [3]. All these conditions restrict the motion of leaflets and obstruct the blood flow into the left ventricle during diastole causing mitral stenosis or, less commonly, mitral regurgitation. Usually, the anomaly is detected in infants and young children; adult presentation is extremely rare and is generally mild in terms of mitral stenosis [4].

\section{Case Report}

We report the case of 27-year-old man affected by Hodgkin's lymphoma-mixed cellularity subtype with polychemotherapy treatment and Barrett's esophagus who came to our attention for routine echocardiographic exam and evaluation of left ventricular function. The patient did not report any dyspnea. In the presence of normal left ventricular systolic function and mildmoderate mitral regurgitation (Fig. 1), a careful examination of mitral apparatus showed a single papillary muscle, unusually located between anterolateral and posteromedial papillary muscles normal position, larger than normal: the aspect was extremely suspect for PMV (Fig. 1b). Mild mitral valve prolapse was also present. Indexed left ventricular end-diastolic volume was $68 \mathrm{ml} / \mathrm{m}^{2}$, left ventricular ejection fraction $60 \%$, and

This is an open-access article distributed under the terms of the Creative Commons Attribution-NonCommercial 4.0 International License, which permits unrestricted use, distribution, and reproduction in any medium for non-commercial purposes, provided the original author and source are credited, a link to the CC License is provided, and changes - if any - are indicated. 


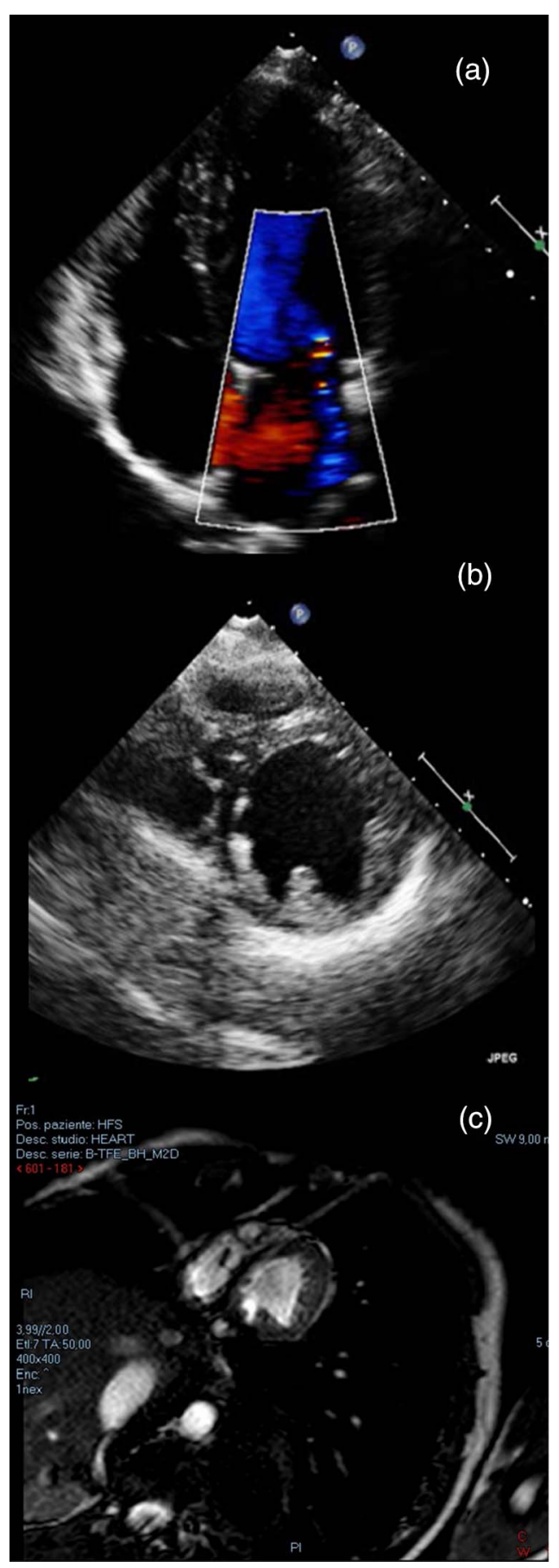

Fig. 1. (a) Mild-moderate mitral regurgitation with asymmetric jet. (b) Single papillary muscle located between anterolateral and posteromedial papillary muscles usual position. (c) Cardiac magnetic resonance confirming the presence of a single papillary muscle

left atrium diameter $41 \mathrm{~mm}$ (anteroposterior diameter). For further assessment, the patient was referred for cardiac magnetic resonance (CMR) because of the presence of Barrett's esophagus, which confirmed the diagnosis of PMV and excluded other associated congenital anomalies (Fig. Ic). No sign of aortic coarctation was found.

\section{Discussion}

PMV is a rare cardiac congenital malformation that is typically diagnosed in childhood and not unusually associated with other congenital malformations and obstruction of left chambers; this condition, known as Shone's syndrome, includes supravalvular ring of the left atrium, subaortic stenosis, and coarctation of the aorta. PMV could be associated with other congenital malformations with different rates: aortic coarctation (68\%), atrial septal defect $(54 \%)$, ventricular septal defect $(46 \%)$, aortic valve stenosis $(32 \%)$, subaortic stenosis $(20 \%)$, and left ventricular hypoplasia (19\%) [5]. The finding of PMV in adults is rare and often following the clinical diagnosis to mitral stenosis or regurgitation; sometimes, the diagnosis could be an occasional finding.

Rim et al. showed that PMV may severely wrinkle leaflets narrowing interchordal spaces at peak systole, compared to those of the normal MV. Furthermore, there is a complex leaflet coaptation with flatter leaflets deformation and a smaller orifice-to-annulus ratio [6]. All these abnormalities are the cause of mitral stenosis and/or regurgitation in patients with PMV.

In our clinical case, the presence of mitral regurgitation without stenosis is probably the expression of the particular position of papillary muscle that was located closer to ventricular apex than normal; therefore, more elongated tendinous chordae permit a greater excursion of mitral leaflets without valve stenosis.

The diagnosis of this rare congenital malformation is generally suspected with transthoracic echocardiography and confirmed by transesophageal echocardiogram. In our case, CMR was extremely useful for the confirmation of PMV, given the presence of Barrett's esophagus that usually contraindicates invasive transesophageal examinations. Nevertheless, even in the absence of any contraindication to invasive examinations, CMR may add further information on ventricular dimension, systolic function, degree of mitral regurgitation, and other possible associated congenital abnormalities.

\section{Conclusion}

Despite the rare incidence of PMV and a diagnosis generally based on transesophageal echocardiography, CMR could represent a useful tool, in selected cases, to confirm the diagnosis of PMV, identify other possible congenital anomalies, and assess ventricular and valvular function.

Funding sources: None.

Authors' contribution: All authors have read and approved the final version of the manuscript.

Conflict of interest: The authors have no potential conflict of interest to disclose. 
Acknowledgements: This work was performed at the University of Foggia, Department of Cardiology, Foggia, Italy.

\section{References}

1. Shone JD, Sellers RD, Anderson RC, Adams P, Lillehei CW, Edwards JE: The developmental complex of "parachute mitral valve," supravalvular ring of left atrium, subaortic stenosis, and coarctation of aorta. Am J Cardiol 11, 714-725 (1963)

2. Rosenquist GC: Congenital mitral valve disease associated with coarctation of the aorta: A spectrum that includes parachute deformity of the mitral valve. Circulation 49, 985-993 (1974)
3. Oosthoek PW, Wenink AC, Macedo AJ, Gittenberger-de Groot AC: The parachute-like asymmetric mitral valve and its two papillary muscles. J Thorac Cardiovasc Surg 114, 9-15 (1997)

4. López-Pardo F, Urbano-Moral JA, González-Calle A, LavianaMartinez F, Esteve-Ruiz I, Lagos-Degrande O, López-Haldon JE: Three-dimensional transesophageal echocardiography in the anatomical assessment of isolated parachute mitral valve in an adult patient. Echocardiography 32, 1732-1735 (2015)

5. Fitzsimons B, Koch CG: Parachute mitral valve. Anesth Analg 101, 1613-1614 (2005)

6. Rim Y, McPherson DD, Kim H: Effect of congenital anomalies of the papillary muscles on mitral valve function. J Med Biol Eng 35, 104-112 (2015) 